\title{
Variational models for phase separation
}

\author{
MARGHERITA SOLCI ${ }^{\dagger}$ AND ENRICO VITALI ${ }^{*}$ \\ Dipartimento di Matematica, Università di Pavia, \\ via Ferrata 1, 27100 Pavia, Italy
}

[Received 25 January 2002]

\begin{abstract}
The paper deals with the asymptotic behaviour (as $\varepsilon \rightarrow 0$ ) of a family $F_{\varepsilon}(u, v)$ of integral functionals in the framework of phase separation. In order to obtain a selection criterion for the minima of the usual double-well, non-convex free energy involving the phase-variable $u$, we add a gradient term in a new variable $v$ which is related to $u$ through the $L^{2}$-distance between $u$ and $v$, weighted by a coefficient $\alpha$. We prove that the limit as $\varepsilon \rightarrow 0$ is a minimal area model with a surface tension of non-local form. The well-known Modica-Mortola constant can be recovered in this setting as a limit case when $\alpha \rightarrow+\infty$.
\end{abstract}

Keywords: Phase separation; non-local models; variational methods; $\Gamma$-convergence.

\section{Introduction}

In this paper we study the asymptotic behaviour of a family of integral functionals, showing their close connection with classical models for phase separation.

A material, e.g. a fluid, which may be in either of two phases, fills a bounded regular region $\Omega$ in space. A configuration of the system can be described by a function on $\Omega$ which takes value 0 on the subset of $\Omega$ occupied by one phase, and value 1 where the material is in the other phase. We could also think of $u$ as the density of the fluid, taking either of two different values $a$ and $b$.

A physically reasonable criterion requires that the interface between the two phases at the equilibrium has minimal area. This corresponds to postulating a free energy of the form

$$
F(u)=\sigma \mathcal{H}^{2}(S(u)),
$$

where $\sigma$ is the surface tension, $S(u)$ is the discontinuity set of $u$ and $\mathcal{H}^{2}$ denotes the 2-dimensional surface measure. The minimization of $F$ is considered among all admissible configurations $u$.

A different approach assumes that the free energy, per unit volume, is a prescribed function $W$ of the density distribution $u$. Thus the stable configurations of the fluid minimize the total energy

$$
E(u)=\int_{\Omega} W(u(x)) \mathrm{d} x,
$$

under the constraint of prescribed total mass: $\int_{\Omega} u(x) \mathrm{d} x=m$. Assume $W$ is of "double-well" type, with minima located at $u=a$ and $u=b$. The addition of an affine function to $W$ does not change the minimum problem because of the condition $\int_{\Omega} u(x) \mathrm{d} x=m$; thus we can assume the same level for both the minima at $a$ and $b$.

\footnotetext{
† Email: marghe@dimat.unipv.it
}

‡Email: vitali@dimat.unipv.it 
If $a|\Omega|<m<b|\Omega|$, the minimum problem for $E(u)$ admits infinitely many piecewise constant solutions with values $a$ and $b$ (see, e.g., [10]), without any condition on the interface between the sets $\{u=a\}$ and $\{u=b\}$ corresponding to the phases. A modification of the model therefore is needed to select some sort of "physically preferred" solutions. The Van der Waals and CahnHilliard approach consists in the addition of a penalty on the density gradient, thus considering the total energy

$$
G_{\varepsilon}(u)=\int_{\Omega}\left(W(u)+\varepsilon^{2}|\nabla u|^{2}\right) \mathrm{d} x,
$$

where $\varepsilon>0$ is a small parameter. A link between the two models can be established via the asymptotic analysis for $\varepsilon \rightarrow 0$. More precisely, a suitable rescaling $\lambda_{\varepsilon} G_{\varepsilon}$ (which admits the same minimizers) is needed to give rise to a non-trivial limit functional. In [17] (see also [18]) Modica proved that, under the constraint of prescribed mass, the $\Gamma$-limit of

$$
F_{\varepsilon}(u)=\frac{1}{\varepsilon} G_{\varepsilon}(u)=\frac{1}{\varepsilon} \int_{\Omega} W(u)+\varepsilon \int_{\Omega}|\nabla u|^{2}
$$

is finite only if $u$ is a function of bounded variation and $u \in\{a, b\}$ a.e., and in that case it is given by (1.1), with

$$
\sigma=2 \int_{a}^{b} \sqrt{W(s)} \mathrm{d} s=\inf \left\{\int_{\mathbb{R}}\left(W(u)+\left(u^{\prime}\right)^{2}\right): \lim _{t \rightarrow-\infty} u(t)=a, \lim _{t \rightarrow+\infty} u(t)=b\right\} .
$$

A variant proposed for the Cahn-Hilliard functional $F_{\varepsilon}$ reads as follows (see [15], [1], [2]):

$$
E_{\varepsilon}(u)=\frac{1}{\varepsilon} \int_{\Omega} W(u(x)) \mathrm{d} x+\frac{1}{4 \varepsilon^{2}} \iint_{\Omega \times \Omega} \mathrm{e}^{-|x-y| / \varepsilon}(u(x)-u(y))^{2} \mathrm{~d} x \mathrm{~d} y .
$$

This corresponds to the replacement of the Dirichlet term $\varepsilon^{2} \int_{\Omega}|\nabla u|^{2}$ in $G_{\varepsilon}$ with a non-local term where spatial inhomogeneity, weighted with an influence kernel, is penalized.

In such a case (see [3] for interaction potentials more general than the exponential kernel above) the limit as $\varepsilon \rightarrow 0$ is again the functional 1.1 , but with a different surface tension:

$$
\begin{aligned}
\sigma=\inf \left\{\int_{\mathbb{R}} W(u(t)) \mathrm{d} t+\frac{1}{4} \iint_{\mathbb{R}^{2}} \mathrm{e}^{-|s-t|}(u(t)-u(s))^{2} \mathrm{~d} t \mathrm{~d} s:\right. \\
\left.\qquad \lim _{t \rightarrow-\infty} u(t)=a, \lim _{t \rightarrow+\infty} u(t)=b\right\} .
\end{aligned}
$$

In this paper we consider the following generalization of $F_{\varepsilon}$ :

$$
F_{\varepsilon}(u, v)=\frac{1}{\varepsilon} \int_{\Omega} W(u)+\frac{\alpha}{\varepsilon} \int_{\Omega}(u-v)^{2}+\varepsilon \int_{\Omega}|\nabla v|^{2},
$$

where $\alpha$ is a positive parameter. Let us notice that in the one-dimensional setting these functionals were proposed by Rogers and Truskinovsky in [21] (see also [9]) as a model for the longitudinal deformation of an elastic bar which could take into account an elastic energy of Ericksen's type together with an internal scalar variable which measures the deviation from one-dimensional deformation. 
We shall show that the limit functional $F(u, v)$ is finite if and only if $u$ is a function of bounded variation and $u=v \in\{a, b\}$ a.e., and again it is given by (1.1), with a surface tension of type (**) (here we assume $a=0$ and $b=1$ ):

$$
\begin{aligned}
\sigma=\sigma(\alpha)=\sqrt{\alpha} \inf \left\{\int_{\mathbb{R}} W(u)+\frac{\alpha^{2}}{4} \iint_{\mathbb{R}^{2}} \mathrm{e}^{-\alpha|x-y|}(u(x)-u(y))^{2} \mathrm{~d} x \mathrm{~d} y:\right. \\
\left.\lim _{t \rightarrow-\infty} u(t)=0, \lim _{t \rightarrow+\infty} u(t)=1\right\} .
\end{aligned}
$$

However, the surface tension $(*)$ corresponding to the family $F_{\varepsilon}(u)$ in 1.2 will be recovered for $\alpha \rightarrow+\infty$.

Actually, we shall consider, in place of $\int_{\Omega}|\nabla v|^{2}$ in $F_{\varepsilon}(u, v)$, a more general anisotropic term $\int_{\Omega} g^{2}(\nabla v)$, with $g$ positively 1-homogeneous. This will give rise, in the limit, to an anisotropic term of the form $\int_{S(u)} \varphi\left(v_{u}\right) \mathrm{d} \mathcal{H}^{2}$, where $v_{u}$ is a normal unit vector field on $S(u)$.

\section{Notation and preliminaries}

The Lebesgue measure in $\mathbb{R}^{n}$ and the $(n-1)$-dimensional Hausdorff measure will be denoted respectively by $\mathcal{L}^{n}$ and $\mathcal{H}^{n-1}$; we shall also use the notation $|E|$ instead of $\mathcal{L}^{n}(E)$. $B_{r}(x)$ will be the open ball with centre $x$ and radius $r$, and $S^{n-1}$ the boundary of the ball $B_{1}(0)$.

Functions of bounded variation and sets of finite perimeter. For the general theory of this topic we refer to [13, 14, 6]; here we recall some definitions and properties we shall use in what follows.

Given an open subset $\Omega$ of $\mathbb{R}^{n}$, a function $u: \Omega \rightarrow \mathbb{R}$ is said to be of bounded variation $(u \in B V(\Omega))$ if $u \in L^{1}(\Omega)$ and its distributional derivatives $D_{i} u$ are Radon measures with finite total variation in $\Omega$. if

If $u \in L_{\text {loc }}^{1}(\Omega)$ we say that $z \in \mathbb{R}$ is the approximate limit of $u$ in $x \in \Omega\left(z=\operatorname{ap}-\lim _{y \rightarrow x} u(y)\right)$

$$
\lim _{\rho \rightarrow 0} \rho^{-n} \int_{B_{\rho}(x)}|u(y)-z| \mathrm{d} y=0 .
$$

The set $S(u)$ of points where this property does not hold is called the approximate discontinuity set of $u$. The set $S(u)$ is a Borel set, and $|S(u)|=0$. If $u \in B V(\Omega)$, then $S(u)$ is countably $(n-1)$ rectifiable, and there exist Borel functions $v_{u}: S(u) \rightarrow S^{n-1}$ and $u^{+}, u^{-}: S(u) \rightarrow \mathbb{R}$ such that for $\mathcal{H}^{n-1}$-a.e. $x \in S(u)$,

$$
\lim _{\rho \rightarrow 0} \rho^{-n} \int_{B_{\rho}^{+}(x) \cap \Omega}\left|u(y)-u^{+}(x)\right| \mathrm{d} y=0, \quad \lim _{\rho \rightarrow 0} \rho^{-n} \int_{B_{\rho}^{-}(x) \cap \Omega}\left|u(y)-u^{-}(x)\right| \mathrm{d} y=0,
$$

where $B_{\rho}^{+}(x)=\left\{y \in B_{\rho}(x):\left\langle y-x, v_{u}(x)\right\rangle>0\right\}$ and $B_{\rho}^{-}(x)=\left\{y \in B_{\rho}(x):\left\langle y-x, v_{u}(x)\right\rangle<0\right\}$.

If $u \in B V(\Omega)$ we denote by $\nabla u$ the density of the absolutely continuous part $D^{a} u$ of the vector measure $D u$ with respect to the Lebesgue measure. Let $D^{s} u$ be the singular part of $D u$, and define $D^{j} u=D u\left\llcorner S(u)\right.$ and $D^{c} u=D u\llcorner(\Omega \backslash S(u)$ ) (the jump and Cantor part of $D u$, respectively). We say that $u$ is a special function of bounded variation $(u \in S B V(\Omega))$ if $D^{c} u=0$; in that case the following decomposition of $D u$ holds:

$$
D u=\nabla u \mathcal{L}^{n}+\left(u^{+}-u^{-}\right) v_{u} \mathcal{H}^{n-1}\llcorner S(u) .
$$


If $E$ is a Borel subset of $\mathbb{R}^{n}$, the essential boundary $\partial^{*} E$ of $E$ is defined as

$$
\partial^{*} E=\left\{x \in \mathbb{R}^{n}: \limsup _{\rho \rightarrow 0} \rho^{-n}\left|B_{\rho}(x) \cap E\right|>0, \limsup _{\rho \rightarrow 0} \rho^{-n}\left|B_{\rho}(x) \backslash E\right|>0\right\} .
$$

It turns out that the discontinuity set of the characteristic function $\chi_{E}$ coincides with $\partial^{*} E$, i.e.

$$
S\left(\chi_{E}\right)=\partial^{*} E .
$$

It can be proved (see [14]) that for any open subset $\Omega$ of $\mathbb{R}^{n}$,

$$
\int_{\Omega}\left|D \chi_{E}\right|=\mathcal{H}^{n-1}\left(\Omega \cap \partial^{*} E\right) .
$$

In particular, if $E$ is a bounded Borel subset, then $\chi_{E} \in B V(\Omega)$ if and only if $\mathcal{H}^{n-1}\left(\Omega \cap \partial^{*} E\right)<$ $+\infty$ (in such a case, $E$ is said to have finite perimeter in $\Omega$ ).

Supremum of a family of measures. Taking into account the regularity of positive Borel measures and the standard properties of the least upper bound of a family of measures, we get the following result (see, e.g., [8, Proposition 1.16]):

Proposition 1.1 Let $\Omega$ be an open subset of $\mathbb{R}^{n}$ and $\mathcal{F}$ a finite, positive set function defined on the family of open subsets of $\Omega$. Let $\mu$ be a positive Borel measure on $\Omega$, and $\left(f_{i}\right)_{i \in I}$ a family of positive Borel functions on $\Omega$. Assume that $\mathcal{F}(A) \geqslant \int_{A} f_{i} \mathrm{~d} \mu$ for all $A$ and $i$, and that $\mathcal{F}(A \cup B) \geqslant$ $\mathcal{F}(A)+\mathcal{F}(B)$ whenever $A, B \subset \subset \Omega$ and $\bar{A} \cap \bar{B}=\emptyset$ (superadditivity). Then $\mathcal{F}(A) \geqslant \int_{A}\left(\sup _{i} f_{i}\right) \mathrm{d} \mu$ for every $A$.

$\Gamma$-convergence. We recall the notion of $\Gamma$-convergence (we refer to [11] for a complete analysis of the subject). Let $(X, d)$ be a metric space, $F_{\varepsilon}: X \rightarrow \overline{\mathbb{R}}(\varepsilon>0)$ a family of functionals, and $F: X \rightarrow \overline{\mathbb{R}}$. We say that $\left\{F_{\varepsilon}\right\} \Gamma$-converges to $F$ at $x \in X$ as $\varepsilon \rightarrow 0$ if:

(i) for every infinitesimal sequence $\left\{\varepsilon_{j}\right\}$ and for every sequence $\left\{x_{j}\right\}$ converging to $x$ in $X$, we have $F(x) \leqslant \liminf _{j \rightarrow \infty} F_{\varepsilon_{j}}\left(x_{j}\right)$;

(ii) for every infinitesimal sequence $\left\{\varepsilon_{j}\right\}$ there exists a sequence $\left\{x_{j}\right\}$ converging to $x$ in $X$ such that $F(x)=\lim _{j \rightarrow \infty} F_{\varepsilon_{j}}\left(x_{j}\right)$

If (i) and (ii) hold for every $x \in X$ we say that $\left\{F_{\varepsilon}\right\} \Gamma$-converges to $F$ in $X$, and write $F=$ $\Gamma-\lim _{\varepsilon \rightarrow 0} F_{\varepsilon}$.

REMARK 1.2 (a) Condition (ii) in the previous definition can be replaced by

(ii) $^{\prime}$ for every $\eta>0$ and for every infinitesimal sequence $\left\{\varepsilon_{j}\right\}$ there exists a sequence $\left\{x_{j}\right\}$ converging to $x$ in $X$ such that $F(x) \geqslant \limsup _{j \rightarrow \infty} F_{\varepsilon_{j}}\left(x_{j}\right)-\eta$.

(b) If $F_{\varepsilon}=G$ is constant with respect to $\varepsilon$, the $\Gamma$-limit exists and coincides with the lower semicontinuous envelope of $G$ with respect to the topology induced by $d$.

REMARK 1.3 The $\Gamma$-lower limit and the $\Gamma$-upper limit of $\left\{F_{\varepsilon}\right\}$ are defined as follows:

$$
\begin{aligned}
F^{\prime}(x) & =\inf \left\{\liminf _{j \rightarrow \infty} F_{\varepsilon_{j}}\left(x_{j}\right): \varepsilon_{j} \rightarrow 0, x_{j} \rightarrow x\right\}, \\
F^{\prime \prime}(x) & =\inf \left\{\limsup _{j \rightarrow \infty} F_{\varepsilon_{j}}\left(x_{j}\right): \varepsilon_{j} \rightarrow 0, x_{j} \rightarrow x\right\} .
\end{aligned}
$$

It is not difficult to see that $F^{\prime}$ and $F^{\prime \prime}$ are both lower semicontinuous and that $\left\{F_{\varepsilon}\right\} \Gamma$-converges if and only if $F^{\prime}=F^{\prime \prime}$. 


\section{Setting of the problem and statement of the results}

Let $n \geqslant 1$ be fixed. Let $W$ and $g$ be given functions satisfying the following conditions:

$-W: \mathbb{R} \rightarrow[0,+\infty)$ belongs to $C^{0}(\mathbb{R})$ and $W(t)=0$ if and only if $t \in\{0,1\}$; moreover $W$ has at least linear growth at $\pm \infty$;

- $g: \mathbb{R}^{n} \rightarrow[0,+\infty)$ is Lipschitz continuous and positively 1-homogeneous, and $g(\xi)>0$ if $\xi \neq 0$. Moreover, we assume that $g(-\xi)=g(\xi)$ for every $\xi$.

For every open subset $\Omega$ of $\mathbb{R}^{n}$ and for every $\varepsilon>0$, we define $F_{\varepsilon}(\cdot, \cdot ; \Omega):\left[L^{1}(\Omega)\right]^{2} \rightarrow[0,+\infty]$ as follows:

$$
F_{\varepsilon}(u, v ; \Omega)= \begin{cases}\frac{1}{\varepsilon} \int_{\Omega} W(u)+\frac{\alpha}{\varepsilon} \int_{\Omega}(u-v)^{2}+\varepsilon \int_{\Omega} g^{2}(D v) & \text { if } v \in H^{1}(\Omega), \\ +\infty & \text { otherwise }\end{cases}
$$

where $\alpha$ is a given positive constant. Moreover, for every measurable $\varphi: \mathbb{R} \rightarrow \mathbb{R}$ and $\psi \in H_{\text {loc }}^{1}(\mathbb{R})$ let

$$
\begin{aligned}
\mathcal{F}^{\alpha}(\varphi, \psi) & =\int_{\mathbb{R}} W(\varphi)+\alpha \int_{\mathbb{R}}(\varphi-\psi)^{2}+\int_{\mathbb{R}}\left(\psi^{\prime}\right)^{2} \\
\mathcal{E}^{\alpha}(\varphi) & =\int_{\mathbb{R}} W(\varphi)+\frac{\alpha^{2}}{4} \iint_{\mathbb{R}^{2}} \mathrm{e}^{-\alpha|x-y|}(\varphi(x)-\varphi(y))^{2} \mathrm{~d} x \mathrm{~d} y .
\end{aligned}
$$

It will be useful to introduce the following function spaces. Given $a, b \in \mathbb{R}$ and a bounded interval $I \subseteq \mathbb{R}$, we shall denote by $H_{a, b}^{1}(I)$ (respectively $H_{a, b}^{1}(\mathbb{R})$ ) the subset of $H^{1}(I)$ (respectively $H_{\text {loc }}^{1}(\mathbb{R})$ ) whose elements have (limit) boundary values $a, b$. Moreover, we set

$$
X=\left\{\varphi: \mathbb{R} \rightarrow[0,1] \text { measurable such that } \lim _{x \rightarrow-\infty} \varphi(x)=0, \lim _{x \rightarrow+\infty} \varphi(x)=1\right\} .
$$

In this paper we prove the following theorem about the asymptotic behaviour of $\left\{F_{\varepsilon}\right\}$ as $\varepsilon \rightarrow 0$ :

THEOREM 2.1 For every bounded open subset $\Omega$ of $\mathbb{R}^{n}$ the functionals $F_{\varepsilon}(\cdot, \cdot ; \Omega) \Gamma$-converge, in $\left[L^{1}(\Omega)\right]^{2}$ as $\varepsilon \rightarrow 0$, to the functional $F(\cdot, \cdot ; \Omega):\left[L^{1}(\Omega)\right]^{2} \rightarrow[0,+\infty]$ defined by

$$
F(u, v ; \Omega)= \begin{cases}c_{W}(\alpha) \int_{S(u)} g^{* *}\left(v_{u}\right) \mathrm{d} \mathcal{H}^{n-1} & \text { if } u, v \in B V(\Omega) \text { and } u=v \in\{0,1\} \text { a.e. } \\ +\infty & \text { otherwise }\end{cases}
$$

where $g^{* *}$ denotes the convex hull of $g$, and

$$
c_{W}(\alpha)=\sqrt{\alpha} \inf \left\{\mathcal{E}^{\alpha}(\varphi): \varphi \in X\right\}
$$

Furthermore, we shall look into the behaviour of the surface tension term $c_{W}(\alpha)$, recovering, in the limit as $\alpha \rightarrow+\infty$, the well-known Modica-Mortola constant ([17], [18]). More precisely we prove (see $\$ 5)$ :

PROPOSITION 2.2 (a) The following representation holds:

$$
c_{W}(\alpha)=\inf \left\{\mathcal{F}^{\alpha}(\varphi, \psi): \varphi \in X, \psi \in H_{0,1}^{1}(\mathbb{R}), \varphi, \psi \text { increasing }\right\} .
$$

Moreover, the infimum on the right-hand side is attained. 
(b) The map $c_{W}: \alpha \mapsto c_{W}(\alpha)$ is strictly increasing, and

$$
\lim _{\alpha \rightarrow+\infty} c_{W}(\alpha)=\min \left\{\int_{\mathbb{R}} W(\varphi)+\int_{\mathbb{R}}\left(\varphi^{\prime}\right)^{2}: \varphi \in H_{0,1}^{1}(\mathbb{R})\right\} .
$$

REMARK 2.3 (a) One can easily see that it is enough to establish Theorem 2.1 when $\alpha=1$, the general case following with $W$ and $g$ replaced by $W / \alpha$ and $g / \sqrt{\alpha}$, respectively.

(b) The proof of Theorem 2.1 relies on the study of functionals obtained by suitable restrictions of $F_{\varepsilon}$ to parallel lines ("slicing method"). Thus, we consider the 1-dimensional case first, taking the following family $F_{\varepsilon}^{\beta}(\cdot, \cdot ; I):\left[L^{1}(I)\right]^{2} \rightarrow[0,+\infty]$ into account:

$$
F_{\varepsilon}^{\beta}(u, v ; I)= \begin{cases}\frac{1}{\varepsilon} \int_{I} W(u)+\frac{1}{\varepsilon} \int_{I}(u-v)^{2}+\varepsilon \beta^{2} \int_{I}\left(v^{\prime}\right)^{2} & \text { if } v \in H^{1}(I), \\ +\infty & \text { otherwise }\end{cases}
$$

where $I \subseteq \mathbb{R}$ is an open set and $\beta>0$. Notice that for every $\sigma>0$,

$$
F_{\varepsilon / \sigma}^{\beta}=\sigma F_{\varepsilon}^{\beta / \sigma}
$$

in particular

$$
F_{\varepsilon / \beta}^{\beta}=\beta F_{\varepsilon}^{1} .
$$

Therefore, the proof will come down to the case $\beta=1$.

\section{The 1-dimensional case}

As noticed in Remark 2.3, the asymptotic behaviour of $\left\{F_{\varepsilon}^{\beta}\right\}$, stated in Corollary 3.2 below, can be immediately deduced from the case $\beta=1$; hence, for every open subset $I$ of $\mathbb{R}$ we shall consider the functionals $F_{\varepsilon}(\cdot, \cdot ; I):\left[L^{1}(I)\right]^{2} \rightarrow[0,+\infty]$ defined by

$$
F_{\varepsilon}(u, v ; I)= \begin{cases}\frac{1}{\varepsilon} \int_{I} W(u)+\frac{1}{\varepsilon} \int_{I}(u-v)^{2}+\varepsilon \int_{I}\left(v^{\prime}\right)^{2} & \text { if } v \in H^{1}(I), \\ +\infty & \text { otherwise. }\end{cases}
$$

THEOREM 3.1 For every open subset $I \subseteq \mathbb{R},\left\{F_{\varepsilon}(\cdot, \cdot ; I)\right\} \Gamma$-converges, in $\left[L^{1}(I)\right]^{2}$ as $\varepsilon \rightarrow 0$, to the functional $F:\left[L^{1}(I)\right]^{2} \rightarrow[0,+\infty]$ defined by

$$
F(u, v ; I)= \begin{cases}c \# S(u) & \text { if } u, v \in B V(I), u=v \text { and } u \in\{0,1\} \text { a.e. } \\ +\infty & \text { otherwise }\end{cases}
$$

where

$$
\begin{aligned}
c & =\inf \left\{\mathcal{E}^{1}(\varphi): \varphi \in X\right\} \\
& =\inf \left\{\int_{\mathbb{R}} W(\varphi)+\frac{1}{4} \iint_{\mathbb{R}^{2}} e^{-|x-y|}(\varphi(x)-\varphi(y))^{2} \mathrm{~d} x \mathrm{~d} y: \varphi \in X\right\} .
\end{aligned}
$$

COROllary 3.2 Let $F_{\varepsilon}^{\beta}$ be as in Remark 2.3 (b). Then for every open subset $I \subseteq \mathbb{R},\left\{F_{\varepsilon}^{\beta}(\cdot, \cdot ; I)\right\}$ $\Gamma$-converges, in $\left[L^{1}(I)\right]^{2}$ as $\varepsilon \rightarrow 0$, to $\beta F(\cdot, \cdot ; I)$, where $F$ is defined in Theorem 3.1 .

In the proof of Theorem 3.1 it will be enough to consider the case $I=(-1,1)$. The extension to an arbitrary interval $(a, b)$ is immediate since $F_{\varepsilon}(u, v,(a, b))=F_{\sigma}(\varphi, \psi,(-1,1))$ with $\sigma=\frac{2 \varepsilon}{b-a}$, 
$u(x)=\varphi\left(\frac{2 x-a-b}{b-a}\right)$ and $v(x)=\psi\left(\frac{2 x-a-b}{b-a}\right)$. Moreover, it is not difficult to see that the $\Gamma$ - liminf inequality for a single bounded interval implies the same inequality for a disjoint countable union of open intervals (bounded or unbounded), hence for an arbitrary open set (recall property (1.5) for characteristic functions). Finally, the recovery sequence exhibited in the proof of the $\Gamma$-lim sup inequality is obtained by a local argument which naturally extends to the case of a general open set.

The $\Gamma$ - lim inf and $\Gamma$-lim sup inequalities will be proved in Proposition 3.3 and 3.6 respectively, when $I=(-1,1)($ recall Remark 1.2$)$. We shall refer to $F_{\varepsilon}(u, v ;(-1,1))$ simply as $F_{\varepsilon}(u, v)$.

\subsection{The $\Gamma$-lower limit}

This section is devoted to proving the following:

Proposition 3.3 Let $\left\{\varepsilon_{j}\right\}$ be a positive infinitesimal sequence. Then

$$
\liminf _{j \rightarrow \infty} F_{\varepsilon_{j}}\left(u_{j}, v_{j}\right) \geqslant F(u, v)
$$

whenever $\left\{u_{j}\right\},\left\{v_{j}\right\}$ are sequences converging in $L^{1}(-1,1)$ to $u$ and $v$, respectively.

Let $u, v$ and $\left\{u_{j}\right\},\left\{v_{j}\right\}$ be as in the statement. We can suppose that the left-hand side in $(3.2)$ is finite and is a limit; therefore we can also assume that $\sup _{j} F_{\varepsilon_{j}}\left(u_{j}, v_{j}\right)=k<+\infty$, and that $\left\{u_{j}\right\},\left\{v_{j}\right\}$ also converge a.e. Since $F_{\varepsilon_{j}}\left(u_{j}, v_{j}\right) \leqslant k$ for every $j$, an application of the Fatou Lemma gives

$$
\int_{-1}^{1}\left(W(u)+(u-v)^{2}\right) \mathrm{d} x \leqslant \liminf _{j \rightarrow \infty} \int_{-1}^{1}\left(W\left(u_{j}\right)+\left(u_{j}-v_{j}\right)^{2}\right) \mathrm{d} x \leqslant \lim _{j \rightarrow \infty}\left(\varepsilon_{j} k\right)=0 .
$$

Hence $u \in\{0,1\}$ a.e. and $u=v$ a.e. We can assume $u_{j}, v_{j} \in[0,1]$; indeed, if $\hat{u}_{j}=\left(0 \vee u_{j}\right) \wedge 1$ and $\hat{v}_{j}=\left(0 \vee v_{j}\right) \wedge 1$ then $\left\{\hat{u}_{j}\right\}$ and $\left\{\hat{v}_{j}\right\}$ converge to $u$ and $v$ in $L^{1}$, and $F_{\varepsilon_{j}}\left(\hat{u}_{j}, \hat{v}_{j}\right) \leqslant F_{\varepsilon_{j}}\left(u_{j}, v_{j}\right)$.

If $S(u)=\emptyset$ there is nothing to prove. Let $N \leqslant \# S(u)$, and $\left\{t_{1}, \ldots, t_{N}\right\} \subseteq S(u)$. Then we can find intervals $I_{i}=\left(a_{i}, b_{i}\right), i=1, \ldots, N$, such that $-1<a_{1}<t_{1}<b_{1} \leqslant \cdots \leqslant a_{N}<t_{N}<b_{N}<$ 1 , and, as $j \rightarrow \infty$,

$$
u_{j}\left(a_{i}\right), v_{j}\left(a_{i}\right) \rightarrow u\left(a_{i}\right) \in\{0,1\}, \quad u_{j}\left(b_{i}\right), v_{j}\left(b_{i}\right) \rightarrow u\left(b_{i}\right) \in\{0,1\},
$$

with $u\left(a_{i}\right) \neq u\left(b_{i}\right)$. We have

$$
F_{\varepsilon_{j}}\left(u_{j}, v_{j}\right) \geqslant \sum_{i=1}^{N} F_{\varepsilon_{j}}\left(u_{j}, v_{j}, I_{i}\right)
$$

Now, consider the $i$ th term of the sum; without loss of generality we can suppose $u\left(a_{i}\right)=0$, $u\left(b_{i}\right)=1$; indeed, the following argument is easily adapted to the case $u\left(a_{i}\right)=1, u\left(b_{i}\right)=0$, and gives exactly the same result. For every $j$ define

$$
\tilde{u}_{j}^{i}(x)= \begin{cases}0 & \text { if } x \in\left(-1, a_{i}-\varepsilon_{j}\right), \\ u_{j}\left(a_{i}\right)+\frac{u_{j}\left(a_{i}\right)}{\varepsilon_{j}}\left(x-a_{i}\right) & \text { if } x \in\left[a_{i}-\varepsilon_{j}, a_{i}\right), \\ u_{j}(x) & \text { if } x \in\left[a_{i}, b_{i}\right], \\ u_{j}\left(b_{i}\right)+\frac{1-u_{j}\left(b_{i}\right)}{\varepsilon_{j}}\left(x-b_{i}\right) & \text { if } x \in\left(b_{i}, b_{i}+\varepsilon_{j}\right], \\ 1 & \text { if } x \in\left(b_{i}+\varepsilon_{j}, 1\right),\end{cases}
$$


and analogously $\tilde{v}_{j}^{i}$ (clearly, we can suppose $\varepsilon_{j}<\min \left\{a_{i}+1,1-b_{i}\right\}$ for every $j$ ). It is easy to verify that

$$
F_{\varepsilon_{j}}\left(u_{j}, v_{j}, I_{i}\right)=F_{\varepsilon_{j}}\left(\tilde{u}_{j}^{i}, \tilde{v}_{j}^{i}\right)+o(1)_{j \rightarrow \infty} .
$$

Since $\tilde{u}_{j}^{i}$ and $\tilde{v}_{j}^{i}$ take the boundary values 0 and 1 in a neighbourhood of -1 and 1 , respectively, we have

$$
F_{\varepsilon_{j}}\left(\tilde{u}_{j}^{i}, \tilde{v}_{j}^{i}\right) \geqslant \inf \left\{F_{\varepsilon_{j}}\left(\tilde{u}_{j}^{i}, \psi\right): \psi \in H_{0,1}^{1}(-1,1)\right\}
$$

We want to show that

$$
\inf \left\{F_{\varepsilon_{j}}\left(\tilde{u}_{j}^{i}, \psi\right): \psi \in H_{0,1}^{1}(-1,1)\right\}=E_{\varepsilon_{j}}\left(\tilde{u}_{j}^{i},(-1,1)\right)+o(1)_{j \rightarrow \infty},
$$

where, for every open $I \subseteq \mathbb{R}$ and $\varphi: I \rightarrow \mathbb{R}$ measurable, we define

$$
E_{\varepsilon}(\varphi, I)=\frac{1}{\varepsilon} \int_{I} W(\varphi)+\frac{1}{4 \varepsilon^{2}} \iint_{I \times I} \mathrm{e}^{-|x-y| / \varepsilon}(\varphi(x)-\varphi(y))^{2} \mathrm{~d} x \mathrm{~d} y .
$$

This is the key point of the proof, since it leads to a family of functionals whose asymptotic behaviour is known (a similar procedure has been used, e.g., in [21]). Indeed, the one-dimensional version of a result due to G. Alberti and G. Bellettini reads as follows (see [1, Th. 1.4 and §1.9]):

THEOREM 3.4 For every open interval $I \subseteq \mathbb{R},\left\{E_{\varepsilon}(\cdot ; I)\right\} \Gamma$-converges, in $L^{1}(I)$ as $\varepsilon \rightarrow 0$, to $\widetilde{F}: L^{1}(I) \rightarrow[0,+\infty]$ defined by

$$
\widetilde{F}(u)= \begin{cases}c \# S(u) & \text { if } u \in B V(I) \text { and } u \in\{0,1\} \text { a.e. } \\ +\infty & \text { otherwise. }\end{cases}
$$

where $c$ is as in Theorem 3.1

In order to obtain 3.6, we start by proving the following lemma. Set

$$
K_{\varepsilon}(\varphi, \psi)=\frac{1}{\varepsilon} \int_{-1}^{1}(\varphi-\psi)^{2}+\varepsilon \int_{-1}^{1}\left(\psi^{\prime}\right)^{2}
$$

whenever $\varphi \in L^{2}(-1,1)$ and $\psi \in H^{1}(-1,1)$.

Lemma 3.5 Let $a, b \in \mathbb{R}$ and $0<\delta<1$. Let $\mathcal{A}$ be a subset of $L^{\infty}(-1,1)$ such that $\varphi=a$ in $(-1,-1+\delta)$ and $\varphi=b$ in $(1-\delta, 1)$, whenever $\varphi \in \mathcal{A}$. Assume that $\mathcal{A}$ is bounded in $L^{\infty}(-1,1)$. Then for every $\varepsilon>0$ and $\varphi \in \mathcal{A}$,

$\inf \left\{K_{\varepsilon}(\varphi, \psi): \psi \in H_{a, b}^{1}(-1,1)\right\}=\frac{1}{4 \varepsilon^{2}} \int_{-1}^{1} \int_{-1}^{1} \mathrm{e}^{-|x-y| / \varepsilon}(\varphi(x)-\varphi(y))^{2} \mathrm{~d} x \mathrm{~d} y+\sigma(\varepsilon, \varphi)$

where $\lim _{\varepsilon \rightarrow 0} \sigma(\varepsilon, \varphi)=0$ uniformly with respect to $\varphi \in \mathcal{A}$.

Proof. In what follows we denote by $\sigma(\varepsilon)$ any function $\sigma(\varepsilon, \varphi)$ such that $\lim _{\varepsilon \rightarrow 0} \sigma(\varepsilon, \varphi)=0$ uniformly with respect to $\varphi \in \mathcal{A}$. It is easy to see that the infimum in (3.7) is attained when $\psi$ is the solution of the following Euler equation:

$$
\left\{\begin{array}{l}
\psi(x)-\varepsilon^{2} \psi^{\prime \prime}(x)=\varphi(x), \quad x \in[-1,1] \\
\psi(-1)=a, \quad \psi(1)=b
\end{array}\right.
$$


The solution $\psi$ of $\sqrt{3.8}$ is the sum of $\psi_{o}$, solution of the homogeneous problem with the given boundary conditions, and $\psi_{g}$, solution of the Euler equation with Dirichlet homogeneous conditions; we obtain (see, e.g., [19. pp. 312-315] for the calculation of the Green function):

$$
\begin{aligned}
& \psi_{o}(x)=\frac{1}{\sinh (2 / \varepsilon)}\left(b \sinh \frac{x+1}{\varepsilon}-a \sinh \frac{x-1}{\varepsilon}\right), \\
& \psi_{g}(x)=\frac{1}{2 \varepsilon \sinh (2 / \varepsilon)} \int_{-1}^{1}\left(\cosh \frac{2-|x-y|}{\varepsilon}-\cosh \frac{x+y}{\varepsilon}\right) \varphi(y) \mathrm{d} y .
\end{aligned}
$$

Integrating by parts and taking 3.8 into account, we have

$$
\begin{aligned}
K_{\varepsilon}(\varphi, \psi) & =\frac{1}{\varepsilon} \int_{-1}^{1} \varphi^{2}-\frac{1}{\varepsilon} \int_{-1}^{1}\left(2 \varphi \psi-(\psi)^{2}\right)-\varepsilon \int_{-1}^{1} \psi \psi^{\prime \prime}+\varepsilon\left[\psi \psi^{\prime}\right]_{-1}^{1} \\
& =\frac{1}{\varepsilon} \int_{-1}^{1} \varphi^{2}-\frac{1}{\varepsilon} \int_{-1}^{1} \varphi \psi_{o}-\frac{1}{\varepsilon} \int_{-1}^{1} \varphi \psi_{g}+\varepsilon\left[\psi \psi^{\prime}\right]_{-1}^{1} \\
& =: \mathcal{I}_{1}^{\varepsilon}(\varphi)+\mathcal{I}_{2}^{\varepsilon}(\varphi)+\mathcal{I}_{3}^{\varepsilon}(\varphi)+\mathcal{I}_{4}^{\varepsilon}(\varphi) .
\end{aligned}
$$

A simple computation yields

$$
-\frac{1}{\varepsilon} \int_{-1}^{-1+\delta} \varphi \psi_{o}-\frac{1}{\varepsilon} \int_{1-\delta}^{1} \varphi \psi_{o}=-\frac{a^{2}+b^{2}}{\tanh (2 / \varepsilon)}+\sigma(\varepsilon),
$$

and

$$
\left|\frac{1}{\varepsilon} \int_{-1+\delta}^{1-\delta} \varphi \psi_{o}\right| \leqslant\|\varphi\|_{L^{1}(-1,1)}(|a|+|b|) \frac{\sinh [(2-\delta) / \varepsilon]}{\varepsilon \sinh (2 / \varepsilon)}
$$

Hence

$$
\mathcal{I}_{2}^{\varepsilon}(\varphi)=-\left(a^{2}+b^{2}\right)+\sigma(\varepsilon) .
$$

Now we consider $\mathcal{I}_{3}^{\varepsilon}(\varphi)$. Since $\cosh (s-t)=e^{-t} \sinh s+e^{-s} \cosh t$ and $\cosh (s+t)=$ $\cosh s \cosh t+\sinh s \sinh t$, it turns out that

$$
\begin{aligned}
\mathcal{I}_{3}^{\varepsilon}(\varphi)= & \frac{1}{2 \varepsilon^{2} \sinh (2 / \varepsilon)}\left[\left(\int_{-1}^{1} \varphi(x) \cosh \frac{x}{\varepsilon} \mathrm{d} x\right)^{2}+\left(\int_{-1}^{1} \varphi(x) \sinh \frac{x}{\varepsilon} \mathrm{d} x\right)^{2}\right] \\
& -\frac{1}{2 \varepsilon^{2}} \int_{-1}^{1} \int_{-1}^{1} \varphi(x) \varphi(y) \mathrm{e}^{-|x-y| / \varepsilon} \mathrm{d} x \mathrm{~d} y \\
& -\frac{1}{2 \varepsilon^{2} \sinh (2 / \varepsilon)} \int_{-1}^{1} \int_{-1}^{1} \varphi(x) \varphi(y) \mathrm{e}^{-2 / \varepsilon} \cosh \frac{|x-y|}{\varepsilon} \mathrm{d} x \mathrm{~d} y .
\end{aligned}
$$

It is immediate to see that the last term is infinitesimal with $\varepsilon$ uniformly with respect to $\varphi \in \mathcal{A}$. Consider now the first one; splitting the integral as above, we have:

$$
\begin{gathered}
\int_{-1}^{-1+\delta} \varphi(x) \cosh \frac{x}{\varepsilon} \mathrm{d} x=a \varepsilon\left(\sinh \frac{1}{\varepsilon}-\sinh \frac{1-\delta}{\varepsilon}\right), \\
\left|\int_{-1+\delta}^{1-\delta} \varphi(x) \cosh \frac{x}{\varepsilon} \mathrm{d} x\right| \leqslant\|\varphi\|_{L^{1}(-1,1)} \cosh \frac{1-\delta}{\varepsilon}
\end{gathered}
$$




$$
\int_{1-\delta}^{1} \varphi(x) \cosh \frac{x}{\varepsilon} \mathrm{d} x=b \varepsilon\left(\sinh \frac{1}{\varepsilon}-\sinh \frac{1-\delta}{\varepsilon}\right) .
$$

Therefore

$$
\frac{1}{2 \varepsilon^{2} \sinh (2 / \varepsilon)}\left(\int_{-1}^{1} \varphi(x) \cosh \frac{x}{\varepsilon} \mathrm{d} x\right)^{2}=\frac{1}{4}\left(a^{2}+b^{2}\right)+\sigma(\varepsilon) .
$$

In the same way we compute the analogous quadratic term. Thus we conclude that

$$
\mathcal{I}_{3}^{\varepsilon}(\varphi)=-\frac{1}{2 \varepsilon^{2}} \int_{-1}^{1} \int_{-1}^{1} \mathrm{e}^{-|x-y| / \varepsilon} \varphi(x) \varphi(y) \mathrm{d} x \mathrm{~d} y+\frac{1}{2}\left(a^{2}+b^{2}\right)+\sigma(\varepsilon) .
$$

Finally, we want to show that

$$
\mathcal{I}_{4}^{\varepsilon}(\varphi)=\sigma(\varepsilon)
$$

A straightforward computation gives

$$
\begin{aligned}
{\left[\psi_{o} \psi_{o}^{\prime}\right]_{-1}^{1} } & =\frac{a^{2}+b^{2}}{\varepsilon \tanh (2 / \varepsilon)}-\frac{2 a b}{\sinh (2 / \varepsilon)}, \\
{\left[\psi_{o} \psi_{g}^{\prime}\right]_{-1}^{1} } & =-\frac{1}{\varepsilon^{2} \sinh (2 / \varepsilon)}\left(a \int_{-1}^{1} \varphi(y) \sinh \frac{1-y}{\varepsilon} \mathrm{d} y+b \int_{-1}^{1} \varphi(y) \sinh \frac{1+y}{\varepsilon} \mathrm{d} y\right) .
\end{aligned}
$$

Splitting the integral as above we obtain

$$
\left[\psi_{o} \psi_{g}^{\prime}\right]_{-1}^{1}=-\frac{a^{2}+b^{2}}{\varepsilon \tanh (2 / \varepsilon)}+\sigma(\varepsilon)
$$

Therefore (3.9) follows.

The results just proved allow us to say that

$$
K_{\varepsilon}(\varphi, \psi)=\frac{1}{\varepsilon} \int_{-1}^{1} \varphi^{2}-\frac{1}{2 \varepsilon^{2}} \int_{-1}^{1} \int_{-1}^{1} \mathrm{e}^{-|x-y| / \varepsilon} \varphi(x) \varphi(y) \mathrm{d} x \mathrm{~d} y-\frac{1}{2}\left(a^{2}+b^{2}\right)+\sigma(\varepsilon) .
$$

Notice now that, since $\int_{-1}^{1} \mathrm{e}^{-|x-y| / \varepsilon} \mathrm{d} y=2 \varepsilon\left(1-\mathrm{e}^{-1 / \varepsilon} \cosh \frac{x}{\varepsilon}\right)$,

$$
\begin{aligned}
\frac{1}{4 \varepsilon^{2}} \int_{-1}^{1} \int_{-1}^{1} \mathrm{e}^{-|x-y| / \varepsilon}(\varphi(x)-\varphi(y))^{2} \mathrm{~d} x \mathrm{~d} y= & \frac{1}{\varepsilon} \int_{-1}^{1} \varphi^{2}-\frac{\mathrm{e}^{-1 / \varepsilon}}{\varepsilon} \int_{-1}^{1} \varphi^{2}(x) \cosh \frac{x}{\varepsilon} \mathrm{d} x \\
& -\frac{1}{2 \varepsilon^{2}} \int_{-1}^{1} \int_{-1}^{1} \mathrm{e}^{-|x-y| / \varepsilon} \varphi(x) \varphi(y) \mathrm{d} x \mathrm{~d} y .
\end{aligned}
$$

Recalling (3.10), it is now sufficient to show that

$$
\frac{\mathrm{e}^{-1 / \varepsilon}}{\varepsilon} \int_{-1}^{1} \varphi^{2}(x) \cosh \frac{x}{\varepsilon} \mathrm{d} x-\frac{1}{2}\left(a^{2}+b^{2}\right)=\sigma(\varepsilon) .
$$

This can be easily done splitting the integral in the same way as above. 
Let us go back to the proof of 3.6 ; apply the lemma just proved to the case $\mathcal{A}=\left\{\tilde{u}_{j}^{i}: j \in \mathbb{N}\right\}$. In view of the properties of $\left\{\tilde{u}_{j}^{i}\right\}$, the assumptions on $\mathcal{A}$ are satisfied for a suitable $\delta$ (depending on $i)$, with $a=0$ and $b=1$. Since $F_{\varepsilon_{j}}\left(\tilde{u}_{j}^{i}, \psi\right)=\varepsilon^{-1} \int_{-1}^{1} W\left(\tilde{u}_{j}^{i}\right)+K_{\varepsilon_{j}}\left(\tilde{u}_{j}^{i}, \psi\right)$, we obtain 3.6.

Collecting (3.3)-(3.6), it turns out that

$$
F_{\varepsilon_{j}}\left(u_{j}, v_{j}\right) \geqslant \sum_{i=1}^{N} E_{\varepsilon_{j}}\left(\tilde{u}_{j}^{i},(-1,1)\right)+o(1)_{j \rightarrow \infty} .
$$

Notice now that $\tilde{u}_{j}^{i} \rightarrow \tilde{u}^{i}$ in $L^{1}(-1,1)$, where $\tilde{u}^{i}$ is defined by:

$$
\tilde{u}^{i}(x)= \begin{cases}0 & \text { if } x \in\left(-1, a_{i}\right), \\ u(x) & \text { if } x \in\left(a_{i}, b_{i}\right), \\ 1 & \text { if } x \in\left(b_{i}, 1\right) .\end{cases}
$$

Then Theorem 3.4 (with $I=(-1,1)$ ) yields

$$
\liminf _{j \rightarrow \infty} F_{\varepsilon_{j}}\left(u_{j}, v_{j}\right) \geqslant \sum_{i=1}^{N} \liminf _{j \rightarrow \infty} E_{\varepsilon_{j}}\left(\tilde{u}_{j}^{i},(-1,1)\right) \geqslant \sum_{i=1}^{N} \widetilde{F}\left(\tilde{u}^{i}\right) \geqslant c N .
$$

The left-hand side is finite by assumption, and $N \leqslant \# S(u)$ is arbitrary: it follows that $\# S(u)<+\infty$, therefore $u \in B V(-1,1)$ (recall $[1.5)$ ). Furthermore

$$
\liminf _{j \rightarrow \infty} F_{\varepsilon_{j}}\left(u_{j}, v_{j}\right) \geqslant c \# S(u) .
$$

This implies the required inequality 3.2 .

\subsection{The $\Gamma$-upper limit}

In this subsection we prove the following:

Proposition 3.6 Let $\eta>0$ and $\left\{\varepsilon_{j}\right\}$ be a positive infinitesimal sequence. For every $u, v$ in $L^{1}(-1,1)$ there exist sequences $\left\{u_{j}^{\eta}\right\},\left\{v_{j}^{\eta}\right\}$ converging, in $L^{1}(-1,1)$ as $j \rightarrow \infty$, to $u$ and $v$ respectively, and such that

$$
\limsup _{j \rightarrow \infty} F_{\varepsilon_{j}}\left(u_{j}^{\eta}, v_{j}^{\eta}\right) \leqslant F(u, v)+\eta .
$$

Proof. Notice that if $F(u, v)=+\infty$ or $S(u)=\emptyset$ the inequality 3.11 is obvious. Thus, since the approximation is invariant under translations and reflections with respect to $u=1 / 2$, and modifies $u$ only in a small neighbourhood of $S(u)$, we can deal only with the case $\# S(u)=1$, and it is enough to prove (3.11) for $u=v=\chi_{(0,1)}$, the characteristic function of $(0,1)$. Then $F(u, v)=c=c_{W}(1)$. In view of the representation of $c_{W}(\alpha)$ which will be given in Proposition 5.1 for every $\eta>0$ we can find $\varphi_{\eta} \in X$ and $\psi_{\eta} \in H_{\mathrm{loc}}^{1}(\mathbb{R})$ such that $\varphi_{\eta}=\psi_{\eta}=\chi_{(0,+\infty)}$ on $\mathbb{R} \backslash\left(-T_{\eta}, T_{\eta}\right)$ for some $T_{\eta}>0$, and

$$
\mathcal{F}^{1}\left(\varphi_{\eta}, \psi_{\eta}\right) \leqslant c+\eta \text {. }
$$

Define now $u_{\varepsilon}^{\eta}(x)=\varphi_{\eta}(x / \varepsilon)$ and $v_{\varepsilon}^{\eta}(x)=\psi_{\eta}(x / \varepsilon)$ for every $\varepsilon>0$. Clearly, $\left\{u_{\varepsilon}^{\eta}\right\}$ and $\left\{v_{\varepsilon}^{\eta}\right\}$ converge to $u=\chi_{(0,1)}$ in $L^{1}(-1,1)$. Moreover

$$
F_{\varepsilon}\left(u_{\varepsilon}^{\eta}, v_{\varepsilon}^{\eta}\right)=F_{\varepsilon}\left(u_{\varepsilon}^{\eta}, v_{\varepsilon}^{\eta} ; \mathbb{R}\right)=\mathcal{F}^{1}\left(\varphi_{\eta}, \psi_{\eta}\right) .
$$


Then we have

$$
F(u, v)+\eta \geqslant F_{\varepsilon}\left(u_{\varepsilon}^{\eta}, v_{\varepsilon}^{\eta}\right)
$$

for every $\varepsilon<1 / T_{\eta}$.

\section{The $n$-dimensional case}

In this section we prove Theorem 2.1 in the general case of $\Omega$ an open subset of $\mathbb{R}^{n}$. We assume $\alpha=1$ (recall Remark 2.3 (a)).

For every $u, v \in L^{1}(\Omega)$ and $A$ an open subset of $\Omega$ let

$$
F^{\prime}(u, v ; A)=\inf \left\{\liminf _{j \rightarrow \infty} F_{\varepsilon_{j}}\left(u_{j}, v_{j} ; A\right): \varepsilon_{j} \rightarrow 0, u_{j} \rightarrow u, v_{j} \rightarrow v \text { in } L^{1}(A)\right\}
$$

$\left(\Gamma\right.$-lower limit of $\left.\left\{F_{\varepsilon}(\cdot, \cdot ; A)\right\}\right)$.

Proposition 4.1 For every $u, v \in L^{1}(\Omega)$,

$$
F^{\prime}(u, v ; \Omega) \geqslant F(u, v ; \Omega) .
$$

Proof. The proof follows the steps outlined in [8], where the slicing method is applied to get the lower semicontinuity inequality for $\Gamma$-limits.

Let $E \subseteq \mathbb{R}^{n}$, and $f: E \rightarrow \mathbb{R}$; for every $\xi \in S^{n-1}$ let $E_{\xi}$ be the orthogonal projection of $E$ on $\xi^{\perp}$ (the $(n-1)$-dimensional vector space orthogonal to $\xi$ ); for every $y \in E_{\xi}$ let $E_{\xi, y}=\{t \in \mathbb{R}$ : $y+t \xi \in E\}$, and let $f_{\xi, y}: E_{\xi, y} \rightarrow \mathbb{R}$ be defined by $f_{\xi, y}(t)=f(y+t \xi)$.

We recall the following basic facts (see, e.g., [6, \$3.11]).

(i) Let $u \in B V(\Omega)$ and let $D^{k}$ stand for any of $D^{a}, D^{j}$ or $D^{c}$ (the absolutely continuous, jump or Cantor part of the derivative). Then, for every $\xi \in S^{n-1}$ and for $\mathcal{H}^{n-1}$-a.e. $y \in \Omega_{\xi}$ we have $u_{\xi, y} \in B V\left(\Omega_{\xi, y}\right)$; moreover, if we denote by $\left\langle D^{k} u, \xi\right\rangle$ the component of $D^{k} u$ along $\xi$, then the following representation holds:

$$
\int_{B}\left\langle D^{k} u, \xi\right\rangle=\int_{B_{\xi}} D^{k} u_{\xi, y}\left(B_{\xi, y}\right) \mathrm{d} \mathcal{H}^{n-1}(y)
$$

(ii) Let $u \in L^{1}(\Omega)$; assume that $u_{\xi, y} \in B V\left(\Omega_{\xi, y}\right)$ for every $\xi$ in a basis of $\mathbb{R}^{n}$ and for a.e. $y \in \Omega_{\xi}$, and that

$$
\int_{\Omega_{\xi}}\left|D u_{\xi, y}\right|\left(\Omega_{\xi, y}\right) \mathrm{d} \mathcal{H}^{n-1}(y)<\infty
$$

Then $u \in B V(\Omega)$.

Let $g_{*}$ be the polar function of $g$, i.e.

$$
g_{*}(\xi)=\sup \left\{\frac{\xi \cdot \eta}{g(\eta)}: \eta \in S^{n-1}\right\}, \quad \xi \in \mathbb{R}^{n} .
$$

It is not difficult to see that $\left(g_{*}\right)_{*}$ is the convex hull of $g$. For every open subset $I$ of $\mathbb{R}$ and $\xi \in S^{n-1}$ denote by $\phi_{\varepsilon}^{\xi}$ the functional $F_{\varepsilon}^{\beta}$ of Remark 2.3 (b), with $\beta=1 / g_{*}(\xi)$, i.e.

$$
\phi_{\varepsilon}^{\xi}(\varphi, \psi ; I):= \begin{cases}\frac{1}{\varepsilon} \int_{I} W(\varphi)+\frac{1}{\varepsilon} \int_{I}(\varphi-\psi)^{2}+\frac{\varepsilon}{g_{*}^{2}(\xi)} \int_{I}(\psi)^{\prime 2} & \text { if } \psi \in H^{1}(I), \\ +\infty & \text { otherwise in }\left[L^{1}(I)\right]^{2} .\end{cases}
$$


By Corollary 3.2 the $\Gamma$-limit as $\varepsilon \rightarrow 0$ is

$$
\phi^{\xi}(\varphi, \psi ; I)= \begin{cases}\frac{c}{g_{*}(\xi)} \# S(\varphi) & \text { if } \varphi, \psi \in B V(I), \varphi=\psi \text { and } \varphi \in\{0,1\} \text { a.e. } \\ +\infty & \text { otherwise, }\end{cases}
$$

where $c$ is as in Theorem 3.1 Let

$$
F_{\varepsilon}^{\xi}(u, v ; A)=\int_{A_{\xi}} \phi_{\varepsilon}^{\xi}\left(u_{\xi, y}, v_{\xi, y} ; A_{\xi, y}\right) \mathrm{d} \mathcal{H}^{n-1}(y)
$$

for every open set $A$ of $\Omega$ and for every $u, v \in L^{1}(A)$. An application of Fubini's Theorem shows that if $u, v \in L^{1}(A)$ and $v \in H^{1}(A)$ then

$$
F_{\varepsilon}^{\xi}(u, v ; A)=\frac{1}{\varepsilon} \int_{A} W(u)+\frac{1}{\varepsilon} \int_{A}(u-v)^{2}+\frac{\varepsilon}{g_{*}^{2}(\xi)} \int_{A}|\langle D v, \xi\rangle|^{2} .
$$

Clearly, $F_{\varepsilon}^{\xi} \leqslant F_{\varepsilon}$ (recall that $g(-\eta)=g(\eta)$ for every $\eta$ ). Therefore, by Fatou's Lemma

$$
F^{\prime}(u, v ; A) \geqslant F^{\xi}(u, v ; A),
$$

where

$$
F^{\xi}(u, v ; A)=\int_{A_{\xi}} \phi^{\xi}\left(u_{\xi, y}, v_{\xi, y} ; A_{\xi, y}\right) \mathrm{d} \mathcal{H}^{n-1}(y) .
$$

In view of the explicit form of $\phi^{\xi}$ and by property (ii) above, if $F^{\prime}(u, v ; A)$ is finite, then $u, v \in$ $B V(A), u=v$ and $u \in\{0,1\}$ a.e.; furthermore, by (i),

$$
F^{\xi}(u, v ; A)=c \int_{S(u) \cap A} \frac{\left|\left\langle\xi, v_{u}\right\rangle\right|}{g_{*}(\xi)} \mathrm{d} \mathcal{H}^{n-1} .
$$

Then

$$
F^{\prime}(u, v ; A) \geqslant c \int_{S(u) \cap A} \frac{\left|\left\langle\xi, v_{u}\right\rangle\right|}{g_{*}(\xi)} \mathrm{d} \mathcal{H}^{n-1}
$$

for every open subset $A, \xi \in S^{n-1}$ and $u, v \in L^{1}(A)$. Since the functionals $F_{\varepsilon}$ are local, the set function $\mu(A)=F^{\prime}(u, v ; A)$ is superadditive on disjoint open sets; thus, by Proposition 1.1 .

$$
F^{\prime}(u, v ; A) \geqslant c \int_{S(u) \cap A} \sup _{\xi \in S^{n-1}} \frac{\left|\left\langle\xi, v_{u}\right\rangle\right|}{g_{*}(\xi)} \mathrm{d} \mathcal{H}^{n-1}=c \int_{S(u) \cap A} \sup _{\xi \in S^{n-1}} \frac{\left\langle\xi, v_{u}\right\rangle}{g_{*}(\xi)} \mathrm{d} \mathcal{H}^{n-1} .
$$

The conclusion follows since, as noticed above, the last integrand is the convex hull of $g$.

We now have to estimate the $\Gamma$-upper limit of $\left\{F_{\varepsilon}(\cdot, \cdot ; \Omega)\right\}$, i.e.

$$
F^{\prime \prime}(u, v ; \Omega)=\inf \left\{\limsup _{j \rightarrow \infty} F_{\varepsilon_{j}}\left(u_{j}, v_{j} ; \Omega\right): \varepsilon_{j} \rightarrow 0, u_{j} \rightarrow u, v_{j} \rightarrow v \text { in } L^{1}(\Omega)\right\} .
$$

Let us consider the functional

$$
\mathcal{G}(u)=\int_{S(u)} g\left(v_{u}\right) \mathrm{d} \mathcal{H}^{n-1},
$$


defined for $u \in B V(\Omega ;\{0,1\})$. By general results on functionals defined on partitions (see [4] and [5, in particular §3]) the lower semicontinuous envelope of $\mathcal{G}$ can be written in the form

$$
\overline{\mathcal{G}}(u)=\int_{S(u)} j\left(v_{u}\right) \mathrm{d} \mathcal{H}^{n-1},
$$

where $j$ is the greatest $B V$-elliptic function less than $g$; hence ([5, Example 2.8]) $j$ is convex, so that

$$
\overline{\mathcal{G}}(u) \leqslant \int_{S(u)} g^{* *}\left(v_{u}\right) \mathrm{d} \mathcal{H}^{n-1},
$$

with $g^{* *}$ denoting the convex hull of $g$.

Proposition 4.2 For every $u, v \in L^{1}(\Omega)$,

$$
F^{\prime \prime}(u, v ; \Omega) \leqslant F(u, v ; \Omega) .
$$

Proof. Let $\mathcal{D}$ be the family of characteristic functions of the sets $E=\Omega \cap A$, with $A$ of class $C^{\infty}$ in a neighbourhood of $\Omega$. If $F(u, v ; \Omega)$ is finite, it can be proved that there exist sequences $\left\{u_{k}\right\}$ and $\left\{v_{k}\right\}$ in $\mathcal{D}$ such that $F\left(u_{k}, v_{k} ; \Omega\right) \rightarrow F(u, v ; \Omega)$ (see [8, Proposition 4.7 and Remark 4.8]). By the lower semicontinuity of $F^{\prime \prime}$ it is enough to prove the proposition when $u=v \in \mathcal{D}$.

Let $u=v=\chi_{E} \in \mathcal{D}$. Then $E=\Omega \cap A$, with $A$ a set of class $C^{\infty}$ in a neighbourhood of $\Omega$. Let $\Omega^{\prime}$ be fixed, with $\Omega \subset \subset \Omega^{\prime}$. There exists $\eta_{0}>0$ such that for every $0<\eta<\eta_{0}$, the projection

$$
\pi: E_{\eta}=\{x \in \Omega: \operatorname{dist}(x, \partial A)<\eta\} \rightarrow \partial A \cap \Omega^{\prime}
$$

is well defined. Let $d(x)=\operatorname{dist}(x, \Omega-A)-\operatorname{dist}(x, A)$; it turns out that for $\eta_{0}$ small enough $d$ is smooth in $E_{\eta}$ and $\nu(x)=\nabla d(x)$ is a normal unit vector to $\partial A$ at $\pi(x)$ if $x \in E_{\eta}$.

Let $0<\eta<\eta_{0}$ be fixed and $M=\max \left\{1 / g(\xi): \xi \in S^{n-1}\right\}$. As in the proof of Proposition 3.6. for any fixed $\eta>0$ we can find $\varphi_{\eta} \in X, \psi_{\eta} \in H_{\mathrm{loc}}^{1}(\mathbb{R})$ such that $\varphi_{\eta}=\psi_{\eta}=\chi_{(0,+\infty)}$ on $\mathbb{R} \backslash(-M T, M T)$, for some $T=T_{\eta}>0$, and

$$
\mathcal{F}^{1}\left(\varphi_{\eta}, \psi_{\eta}\right) \leqslant c+\eta
$$

For every $\varepsilon>0$ with $T \varepsilon<\eta$, define $u_{\varepsilon}: \Omega \rightarrow \mathbb{R}$ as follows:

$$
u_{\varepsilon}(x)= \begin{cases}\varphi_{\eta}\left(\frac{d(x)}{\varepsilon g(v(x))}\right) & \text { if }|d(x)| \leqslant T \varepsilon, \\ 0 & \text { otherwise in } \Omega \backslash E, \\ 1 & \text { otherwise in } E .\end{cases}
$$

In the same way we define $v_{\varepsilon}$ on $\Omega$.

Now we want to estimate $F_{\varepsilon}\left(u_{\varepsilon}, v_{\varepsilon} ; \Omega\right)$. Since $d, g, 1 / g, v$ are Lipschitz on $\{|d| \leqslant T \varepsilon\}$, we can find a positive constant $L$ such that

$$
\begin{aligned}
\varepsilon g^{2}\left(D v_{\varepsilon}(x)\right) & =\frac{1}{\varepsilon}\left|\left(\psi_{\eta}\right)^{\prime}\left(\frac{d(x)}{\varepsilon g(v(x))}\right)\right|^{2} g^{2}\left(\frac{\nabla d(x)}{g(v(x))}+d(x) \nabla\left(\frac{1}{g(v(x))}\right)\right) \\
& \leqslant \frac{1}{\varepsilon}\left|\left(\psi_{\eta}\right)^{\prime}\left(\frac{d(x)}{\varepsilon g(v(x))}\right)\right|^{2}\left[g^{2}\left(\frac{v(x)}{g(v(x))}\right)+\varepsilon L\right] \leqslant\left|\left(\psi_{\eta}\right)^{\prime}\left(\frac{d(x)}{\varepsilon g(v(x))}\right)\right|^{2}\left(\frac{1}{\varepsilon}+L\right) .
\end{aligned}
$$


In the following computation we shall drop the subscript $\eta$. Taking the coarea formula into account (see, e.g., [6, \$2.12]), we get

$$
\begin{aligned}
& F_{\varepsilon}\left(u_{\varepsilon}, v_{\varepsilon} ; \Omega\right) \\
& \leqslant\left(\frac{1}{\varepsilon}+L\right) \int_{\Omega \cap\{|d| \leqslant T \varepsilon\}}\left[W\left(\varphi\left(\frac{d(x)}{\varepsilon g(v(x))}\right)\right)+\left(\varphi\left(\frac{d(x)}{\varepsilon g(v(x))}\right)-\psi\left(\frac{d(x)}{\varepsilon g(v(x))}\right)\right)^{2}\right. \\
& \left.+\left|\psi^{\prime}\left(\frac{d(x)}{\varepsilon g(v(x))}\right)\right|^{2}\right] \mathrm{d} x \\
& \leqslant\left(\frac{1}{\varepsilon}+L\right) \int_{-T \varepsilon}^{T \varepsilon} \int_{\{d(x)=t\}}\left[W\left(\varphi\left(\frac{t}{\varepsilon g(v(x))}\right)\right)+\left(\varphi\left(\frac{t}{\varepsilon g(v(x))}\right)-\psi\left(\frac{t}{\varepsilon g(v(x))}\right)\right)^{2}\right. \\
& \left.+\left|\psi^{\prime}\left(\frac{t}{\varepsilon g(v(x))}\right)\right|^{2}\right] \mathrm{d} \mathcal{H}^{n-1}(x) \mathrm{d} t \\
& \leqslant\left(\frac{1}{\varepsilon}+L\right) \int_{-T \varepsilon}^{T \varepsilon} \int_{\partial E \cap \Omega^{\prime}}\left[W\left(\varphi\left(\frac{t}{\varepsilon g(v(y))}\right)\right)+\left(\varphi\left(\frac{t}{\varepsilon g(v(y))}\right)-\psi\left(\frac{t}{\varepsilon g(v(y))}\right)\right)^{2}\right. \\
& \left.+\left|\psi^{\prime}\left(\frac{t}{\varepsilon g(v(y))}\right)\right|^{2}\right] \mathrm{d} \mathcal{H}^{n-1}(y) \mathrm{d} t+o(1)_{\varepsilon \rightarrow 0} \\
& \leqslant(1+\varepsilon L) \int_{-\infty}^{+\infty}\left[W(\varphi(t))+(\varphi(t)-\psi(t))^{2}+\left|\psi^{\prime}(t)\right|^{2}\right] \mathrm{d} t \int_{\partial E \cap \Omega^{\prime}} g(v(y)) \mathrm{d} \mathcal{H}^{n-1}(y)+o(1)_{\varepsilon \rightarrow 0} \\
& \leqslant(1+\varepsilon L)(c+\eta) \int_{\partial E \cap \Omega^{\prime}} g(v(y)) \mathrm{d} \mathcal{H}^{n-1}(y)+o(1)_{\varepsilon \rightarrow 0} .
\end{aligned}
$$

Since $\Omega^{\prime}$ and $\eta$ are arbitrary, we deduce that

$$
F^{\prime \prime}(u, v ; \Omega) \leqslant c \mathcal{G}(u)
$$

The same inequality holds for the relaxed functionals; by the lower semicontinuity of $F^{\prime \prime}$ and by (4.1), we conclude that

$$
F^{\prime \prime}(u, v ; \Omega) \leqslant c \int_{S(u)} g^{* *}\left(v_{u}\right) \mathrm{d} \mathcal{H}^{n-1},
$$

and Proposition 4.2 is completely proved.

\section{Some properties of $c_{W}(\alpha)$}

Proposition 5.1 Let $c_{W}(\alpha)$ be as in Theorem 2.1. Then

$$
\begin{aligned}
c_{W}(\alpha) & =\sqrt{\alpha} \inf \left\{\mathcal{E}^{\alpha}(\varphi): \varphi \in \mathcal{U}\right\} \\
& =\inf \left\{\mathcal{F}^{\alpha}(\varphi, \psi): \varphi \in \mathcal{U}, \psi \in \mathcal{U} \cap H_{\mathrm{loc}}^{1}(\mathbb{R})\right\},
\end{aligned}
$$

where $\mathcal{U}$ is any of the following spaces:

$X$ : the set of measurable $\varphi: \mathbb{R} \rightarrow[0,1]$ such that $\lim _{x \rightarrow-\infty} \varphi(x)=0$ and $\lim _{x \rightarrow+\infty} \varphi(x)=1$; $Y$ : the set of measurable $\varphi: \mathbb{R} \rightarrow[0,1]$ with $\varphi=\chi_{(0,+\infty)}$ on $\mathbb{R} \backslash(-R, R)$ for some $R>0$;

$Z$ : the subset of the increasing elements of $Y$. 
Proof. It is enough to deal with the case $\alpha=1$. Let

$$
I_{\mathcal{U}}=\inf \left\{\mathcal{F}^{1}(\varphi, \psi): \varphi \in \mathcal{U}, \psi \in \mathcal{U} \cap H_{\text {loc }}^{1}(\mathbb{R})\right\}
$$

Clearly, $I_{X} \leqslant I_{Y} \leqslant I_{Z}$.

Step 1. $I_{X}=I_{Y}$.

Let $\sigma>0$ and $\varphi_{\sigma} \in X, \psi_{\sigma} \in X \cap H_{\text {loc }}^{1}(\mathbb{R})$ be such that $\mathcal{F}^{1}\left(\varphi_{\sigma}, \psi_{\sigma}\right) \leqslant I_{X}+\sigma$. There exists $R>0$ with the following property:

$$
\left|\varphi_{\sigma}-\chi_{(0,+\infty)}\right|,\left|\psi_{\sigma}-\chi_{(0,+\infty)}\right|<\sigma \quad \text { on } \mathbb{R} \backslash(-R, R)
$$

Define $\tilde{\psi}_{\sigma}: \mathbb{R} \rightarrow \mathbb{R}$ to be the continuous function which is affine in both the intervals $J=$ $(-R-1,-R), J^{\prime}=(R, R+1)$ and such that

$$
\widetilde{\psi}_{\sigma}=\psi_{\sigma} \quad \text { on }(-R, R), \quad \widetilde{\psi}_{\sigma}=\chi_{(0,+\infty)} \quad \text { on } \mathbb{R} \backslash(-R-1, R+1) .
$$

Moreover, let $\tilde{\varphi}_{\sigma}: \mathbb{R} \rightarrow \mathbb{R}$ be defined by: $\tilde{\varphi}_{\sigma}=\varphi_{\sigma}$ on $\mathbb{R} \backslash(-R, R), \widetilde{\varphi}_{\sigma}=\widetilde{\psi}_{\sigma}$ on $J \cup J^{\prime}$, and $\varphi_{\sigma}=\chi_{(0,+\infty)}$ on $\mathbb{R} \backslash(-R-1, R+1)$. Then $\tilde{\varphi}_{\sigma} \in Y, \widetilde{\psi}_{\sigma} \in Y \cap H_{\mathrm{loc}}^{1}(\mathbb{R})$ and, as one can easily check,

$$
\mathcal{F}^{1}\left(\widetilde{\varphi}_{\sigma}, \widetilde{\psi}_{\sigma}\right) \leqslant \mathcal{F}^{1}\left(\varphi_{\sigma}, \psi_{\sigma}\right)+o(1)_{\sigma \rightarrow 0}
$$

Step 2. $I_{Y}=I_{Z}$.

For every $\varphi \in Y$ and $\psi \in Y \cap H_{\text {loc }}^{1}(\mathbb{R})$ we construct $\bar{\varphi} \in Z$ and $\bar{\psi} \in Z \cap H_{\mathrm{loc}}^{1}(\mathbb{R})$ such that

$$
\mathcal{F}^{1}(\bar{\varphi}, \bar{\psi}) \leqslant \mathcal{F}^{1}(\varphi, \psi) .
$$

Let $R>0$ be such that $\varphi=\psi=\chi_{(0,+\infty)}$ on $\mathbb{R} \backslash(-R, R)$. Following [2], we define the right rearrangement of a subset $A$ of $[-R, R]$ as the set $A^{*}=[R-m(A), R]$. Then, for a measurable function $u:[-R, R] \rightarrow[0,1]$ we define the increasing rearrangement of $u$ as

$$
u^{*}(x)=\sup \left\{c \in \mathbb{R}: x \in I_{c}^{*}\right\}, \quad x \in[-R, R],
$$

where $I_{c}=\{x \in[-R, R]: u(x) \geqslant c\}$. Let

$$
\bar{\varphi}(x)= \begin{cases}0 & \text { if } x<-R, \\ \varphi^{*}(x) & \text { if }-R \leqslant x \leqslant R, \\ 1 & \text { if } x>R,\end{cases}
$$

and let $\bar{\psi}$ be analogously defined. Then $\bar{\varphi} \in Z$ and $\bar{\psi} \in Z \cap H_{\text {loc }}^{1}(\mathbb{R})$; moreover, properties [16, (C), p. 22; (M9), p. 23; (G1a), p. 35] allow us to deduce the inequality (5.1).

Step 3. $\inf \left\{\mathcal{E}^{1}(\varphi): \varphi \in X\right\}=\inf \left\{\mathcal{E}^{1}(\varphi): \varphi \in Y\right\}$.

We only have to prove that the right-hand side does not exceed the left-hand side. Let $\sigma>1$ and $\varphi_{\sigma} \in X$ be such that $\mathcal{E}^{1}\left(\varphi_{\sigma}\right) \leqslant \inf \left\{\mathcal{E}^{1}(\varphi): \varphi \in X\right\} ;$ let $\bar{\varphi}_{\sigma}=\frac{1}{2}+\sigma\left(\varphi_{\sigma}-\frac{1}{2}\right)$ and $\tilde{\varphi}_{\sigma}=\left(\bar{\varphi}_{\sigma} \vee 0\right) \wedge 1$. Then $\tilde{\varphi}_{\sigma} \in Y$, and

$$
\mathcal{E}^{1}\left(\widetilde{\varphi}_{\sigma}\right) \leqslant \mathcal{E}^{1}\left(\bar{\varphi}_{\sigma}\right)=\sigma^{2} \mathcal{E}^{1}\left(\varphi_{\sigma}\right) \leqslant \sigma^{3} \inf \left\{\mathcal{E}^{1}(\varphi): \varphi \in X\right\} .
$$


Step 4. Let $\varphi \in Y$. We claim that

$$
\inf \left\{\mathcal{F}^{1}(\varphi, \psi): \psi \in Y \cap H_{\mathrm{loc}}^{1}(\mathbb{R})\right\}=\mathcal{E}^{1}(\varphi) .
$$

For every $R>0$ let $Y_{R}=\left\{\varphi \in X: \varphi=\chi_{(0,+\infty)}\right.$ on $\left.\mathbb{R} \backslash(-R, R)\right\}$; then $Y=\bigcup_{R>0} Y_{R}$.

By assumption $\varphi \in Y_{R}$ for some $R>0$. If $T>2 R$, let $\varphi_{T}(x)=\varphi(T x)$. Apply Lemma 3.5 with $\mathcal{A}=\left\{\varphi_{T}: T>2 R\right\}$. Then for every $\varepsilon>0$,

$$
\begin{aligned}
\inf \left\{\frac{1}{\varepsilon} \int_{\mathbb{R}}\left(\varphi_{T}-\psi\right)^{2}+\varepsilon \int_{\mathbb{R}}\left(\psi^{\prime}\right)^{2}: \psi\right. & \left.\in H_{0,1}^{1}(\mathbb{R})\right\} \\
& =\frac{1}{4 \varepsilon^{2}} \int_{-1}^{1} \int_{-1}^{1} e^{-|x-y| / \varepsilon}(\varphi(x)-\varphi(y))^{2} \mathrm{~d} x \mathrm{~d} y+\sigma(\varepsilon),
\end{aligned}
$$

with $\lim _{\varepsilon \rightarrow 0} \sigma(\varepsilon)=0$ uniformly with respect to $T>2 R$. Let $\varepsilon=1 / T$ and $\widetilde{\psi}(t)=\psi(t / T)$ whenever $\psi \in H_{0,1}^{1}(-1,1)$; then, by a linear change of variable, we get

$$
\begin{aligned}
\inf \left\{\int_{-T}^{T}(\varphi-\tilde{\psi})^{2}+\varepsilon \int_{-T}^{T}\left(\tilde{\psi}^{\prime}\right)^{2}\right. & : \psi \\
& \left.\in H_{0,1}^{1}(-1,1)\right\} \\
& =\frac{1}{4} \int_{-T}^{T} \int_{-T}^{T} e^{-|t-s|}(\varphi(t)-\varphi(s))^{2} \mathrm{~d} t \mathrm{~d} s+o(1)_{T \rightarrow+\infty},
\end{aligned}
$$

i.e.

$$
\begin{aligned}
\inf \left\{\int_{\mathbb{R}}(\varphi-\psi)^{2}+\varepsilon \int_{\mathbb{R}}\left(\psi^{\prime}\right)^{2}: \psi \in Y_{T}\right. & \left.\cap H_{\mathrm{loc}}^{1}(\mathbb{R})\right\} \\
& =\frac{1}{4} \int_{\mathbb{R}} \int_{\mathbb{R}}^{-|t-s|}(\varphi(t)-\varphi(s))^{2} \mathrm{~d} t \mathrm{~d} s+o(1)_{T \rightarrow+\infty} .
\end{aligned}
$$

It is now enough to notice that

$$
\begin{aligned}
\inf \left\{\mathcal{F}^{1}(\varphi, \psi): \psi\right. & \left.\in Y \cap H_{\mathrm{loc}}^{1}(\mathbb{R})\right\} \\
& =\int_{\mathbb{R}} W(\varphi)+\lim _{T \rightarrow+\infty} \inf \left\{\int_{\mathbb{R}}(\varphi-\psi)^{2}+\varepsilon \int_{\mathbb{R}}\left(\psi^{\prime}\right)^{2}: \psi \in Y_{T} \cap H_{\mathrm{loc}}^{1}(\mathbb{R})\right\} .
\end{aligned}
$$

Step 5. Consider the infimum of both sides of (5.2) when $\varphi$ varies in $Y$; then

$$
I_{Y}=\inf \left\{\mathcal{E}^{1}(\varphi): \varphi \in Y\right\} .
$$

If we restrict $\varphi$ in 5.2 to vary in $Z$, then

$$
\inf \left\{\mathcal{E}^{1}(\varphi): \varphi \in Z\right\}=\inf \left\{\mathcal{F}^{1}(\varphi, \psi): \varphi \in Z, \psi \in Y \cap H_{\mathrm{loc}}^{1}(\mathbb{R})\right\} \leqslant I_{Z}=I_{Y}
$$

We conclude that

$$
\inf \left\{\mathcal{E}^{1}(\varphi): \varphi \in Z\right\}=\inf \left\{\mathcal{E}^{1}(\varphi): \varphi \in Y\right\}
$$

Proof of Proposition 2.2. (a) Let $\left(\varphi_{n}, \psi_{n}\right)$ be a minimizing sequence for $\mathcal{F}^{\alpha}$ on

$$
\widetilde{Z}=\left\{(\varphi, \psi): \varphi \in X, \psi \in X \cap H_{\mathrm{loc}}^{1}(\mathbb{R}), \varphi, \psi \text { increasing }\right\} .
$$


Thanks to the translation invariance of $\mathcal{F}^{\alpha}$, it is not restrictive to suppose that $\varphi_{n} \leqslant 1 / 2$ in $(-\infty, 0)$ and $\varphi_{n} \geqslant 1 / 2$ in $(0,+\infty)$. Since the total variation of the measure $D \varphi_{n}$ is 1 for every $n$, it follows that $\left\{\varphi_{n}\right\}$ is bounded in $B V_{\text {loc }}(\mathbb{R})$; moreover $\psi_{n}^{\prime}$ is bounded in $L^{2}(\mathbb{R})$; by compactness there exist $u_{\alpha} \in B V_{\text {loc }}(\mathbb{R})$ and $v_{\alpha} \in H_{\text {loc }}^{1}(\mathbb{R})$ such that, possibly passing to a subsequence,

$$
\varphi_{n} \rightarrow u_{\alpha} \text { in } L_{\mathrm{loc}}^{1}(\mathbb{R}) \quad \text { and } \quad \psi_{n} \rightarrow v_{\alpha} \text { in } H_{\mathrm{loc}}^{1}(\mathbb{R})
$$

We can also suppose that the convergences are pointwise a.e. By the Fatou Lemma and the weak lower semicontinuity of the norm,

$$
\liminf _{n \rightarrow \infty} \mathcal{F}^{\alpha}\left(\varphi_{n}, \psi_{n}\right) \geqslant \mathcal{F}^{\alpha}\left(u_{\alpha}, v_{\alpha}\right) .
$$

If we prove that $\left(u_{\alpha}, v_{\alpha}\right) \in \widetilde{Z}$, this inequality implies that

$$
\mathcal{F}^{\alpha}\left(u_{\alpha}, v_{\alpha}\right)=\min \left\{\mathcal{F}^{\alpha}(\varphi, \psi):(\varphi, \psi) \in \widetilde{Z}\right\} .
$$

It is clear that $u_{\alpha}$ and $v_{\alpha}$ are increasing; since $\alpha \int_{\mathbb{R}}\left(u_{\alpha}-v_{\alpha}\right)^{2}<+\infty$, we get $\lim _{x \rightarrow+\infty} u_{\alpha}(x)=$ $\lim _{x \rightarrow+\infty} v_{\alpha}(x)$, and the same for $x \rightarrow-\infty$. Then, it is sufficient to prove that $\lim _{x \rightarrow+\infty} u_{\alpha}(x)=1$ and $\lim _{x \rightarrow-\infty} u_{\alpha}(x)=0$. This is an immediate consequence of the fact that $\int_{\mathbb{R}} W\left(\varphi_{\alpha}\right)<+\infty$.

(b) Let

$$
C_{W}=\min \left\{\int_{\mathbb{R}} W(\varphi)+\int_{\mathbb{R}}\left(\varphi^{\prime}\right)^{2}: \varphi \in H_{0,1}^{1}(\mathbb{R})\right\}
$$

We claim that for every $\alpha$,

$$
0<c_{W}(\alpha)<C_{W} .
$$

By (a) we have $c_{W}(\alpha)>0$ for every $\alpha$. To prove that $c_{W}(\alpha)<C_{W}$ we argue by contradiction, assuming that $c_{W}(\alpha) \geqslant C_{W}$. Let $\varphi \in H_{0,1}^{1}(\mathbb{R})$ be such that $\int_{\mathbb{R}} W(\varphi)+\int_{\mathbb{R}}\left(\varphi^{\prime}\right)^{2}=C_{W}$. Given $\zeta \in \mathcal{C}_{c}^{\infty}(\mathbb{R})$ and $t \in \mathbb{R}$, define $\Phi(t)=\mathcal{F}^{\alpha}(\varphi, \varphi+t \zeta)$. Then $\Phi(t) \geqslant c_{W}(\alpha) \geqslant C_{W}=\Phi(0)$ for every $t$. Since $\Phi \in C^{1}(\mathbb{R})$, it follows that

$$
\Phi^{\prime}(0)=2 \int_{\mathbb{R}} u^{\prime} \zeta^{\prime}=0 \quad \forall \zeta \in C_{c}^{\infty}(\mathbb{R}) .
$$

Therefore $\varphi^{\prime \prime}=0$, and this gives a contradiction.

It is immediate to check that the map $\alpha \mapsto c_{W}(\alpha)$ is increasing. We claim that it is strictly increasing. For $\sigma>0$ let $(\varphi, \psi)$ be a solution of the minimum problem dealt with in part (a) for $c_{W}(\alpha+\sigma)$. Then

$$
\begin{aligned}
c_{W}(\alpha+\sigma) & =\int_{\mathbb{R}} W(\varphi)+\alpha \int_{\mathbb{R}}(\varphi-\psi)^{2}+\int_{\mathbb{R}}\left(\psi^{\prime}\right)^{2}+\sigma \int_{\mathbb{R}}(\varphi-\psi)^{2} \\
& \geqslant c_{W}(\alpha)+\sigma \int_{\mathbb{R}}(\varphi-\psi)^{2}
\end{aligned}
$$

if $c_{W}(\alpha)=c_{W}(\alpha+\sigma)$, then $c_{W}(\alpha+\sigma)=C_{W}$, and this gives a contradiction.

Let $\left(\varphi_{\alpha}, \psi_{\alpha}\right)$ be a solution of the minimum problem dealt with in part (a) for $c_{W}(\alpha)$. The translation invariance of the functional allows us to choose $\varphi_{\alpha}$ such that $\varphi_{\alpha} \leqslant 1 / 2$ in $(-\infty, 0)$ 
and $\varphi_{\alpha} \geqslant 1 / 2$ in $(0,+\infty)$. Since $c_{W}(\alpha) \leqslant C_{W}$, weak compactness arguments allow us to deduce that, up to a subsequence:

$$
\begin{aligned}
& \psi_{\alpha} \rightarrow \varphi \quad \text { in } H_{\mathrm{loc}}^{1}(\mathbb{R}) \text { as } \alpha \rightarrow+\infty \\
& \varphi_{\alpha} \rightarrow \varphi \quad \text { in } L_{\mathrm{loc}}^{2}(\mathbb{R}) \text { as } \alpha \rightarrow+\infty
\end{aligned}
$$

Moreover, $\varphi_{\alpha}$ can be assumed to converge pointwise a.e. The Fatou Lemma and the weak lower semicontinuity of the norm yield

$$
\liminf _{\alpha \rightarrow+\infty}\left(\int_{\mathbb{R}} W\left(\varphi_{\alpha}\right)+\alpha \int_{\mathbb{R}}\left(\varphi_{\alpha}-\psi_{\alpha}\right)^{2}+\int_{\mathbb{R}}\left(\psi_{\alpha}^{\prime}\right)^{2}\right) \geqslant \int_{\mathbb{R}} W(\varphi)+\int_{\mathbb{R}}\left(\varphi^{\prime}\right)^{2} .
$$

As in (a) we prove that $\varphi \in H_{0,1}^{1}(\mathbb{R})$. This implies, since $c_{W}(\alpha) \leqslant C_{W}$,

$$
\lim _{\alpha \rightarrow+\infty} c_{W}(\alpha)=C_{W}
$$

thus concluding the proof.

REMARK 5.2 It is easy to check directly that

$$
\lim _{\alpha \rightarrow 0^{+}} c_{W}(\alpha)=0 .
$$

REMARK 5.3 If $W \in C^{3}(\mathbb{R})$, then the infimum in

$$
c_{W}(\alpha)=\inf \left\{\mathcal{E}^{\alpha}(\varphi): \varphi \in X\right\}
$$

is attained when $\varphi$ is a travelling wave solution for the one-dimensional parabolic problem

$$
w_{t}=J * w-w-f(w),
$$

where $f$ is the derivative of $W$ (see [2] p. 549]). The regularity result in [7, Theorem 3.1(c)] allows us to construct a minimizing sequence in $H_{0,1}^{1}(\mathbb{R})$ converging in $L_{\text {loc }}^{1}(\mathbb{R})$ to the minimum point; hence

$$
\min \left\{\mathcal{E}^{\alpha}(\varphi): \varphi \in X\right\}=\inf \left\{\mathcal{E}^{\alpha}(\varphi): \varphi \in H_{0,1}^{1}(\mathbb{R})\right\} .
$$

\section{Acknowledgements}

We are grateful to Prof. Andrea Braides and Prof. Lev Truskinovsky for the fruitful discussions on the subject.

\section{REFERENCES}

1. Alberti, G. \& Bellettini, G. A non-local anisotropic model for phase transitions: asymptotic behaviour of rescaled energies. Euro. J. Appl. Math. 9 (1998), 261-284. MR 99e:80003 Zbl 0932.49018

2. Alberti, G. \& Bellettini, G. A nonlocal anisotropic model for phase transitions. Part I: the Optimal Profile Problem. Math. Ann. 310 (1998), 527-560. MR 2000b:82013 Zbl 0891.49021

3. Alberti, G., Bellettini, G., Cassandro, M., \& Presutti, E. Surface tension in Ising systems with Kac potential. J. Statist. Phys. 82 (1996), 743-796. MR 97b:82044ZZbl 01554034 
4. Ambrosio, L. \& Braides, A. Functionals defined on partitions in sets of finite perimeter I: integral representation and $\Gamma$-convergence. J. Math. Pures Appl. 69 (1990), 285-305. MR 91j:49015 Zbl 0676.49028

5. Ambrosio, L. \& Braides, A. Functionals defined on partitions in sets of finite perimeter II: semicontinuity, relaxation and homogeneization. J. Math. Pures Appl. 69 (1990), 307-333. MR 91j:49016 Zbl 0676.49029

6. Ambrosio, L., Fusco, N., \& Pallara, D. Functions of Bounded Variation and Free Discontinuity Problems. Oxford Math. Monographs, Clarendon Press, Oxford (2000). MR 2003a:49002 Zbl 0957.49001

7. BAtes, P. W., Fife, P. C., REN, X., \& WANG, X. Travelling waves in a convolution model for phase transitions. Arch. Rational Mech. Anal. 138 (1997), 105-136. MR 98f:45004|Zbl 0889.45012

8. BRAides, A. Approximation of Free-Discontinuity Problems. Lecture Notes in Math. 1694, Springer, Berlin (1998). MR 99j:49001 Zbl 0909.49001

9. BRANDOn, D., LIN, T., \& Rogers, R. C. Phase transition and hysteresis in nonlocal and order-parameter models. Meccanica 30 (1995), 541-565. MR 96h:73006 Zbl 0835.73005

10. Carr, J., Gurtin, M. E., \& Slemrod, M. Structured phase transitions on a finite interval. Arch. Rational Mech. Anal. 86 (1984), 317-351. MR 86i:80001 Zbl 0564.76075

11. Dal Maso, G. An Introduction to $\Gamma$-Convergence. Birkhäuser (1993). MR 94a:49001 Zbl 0816.49001|

12. De Giorgi, E. \& Franzoni, T. Su un tipo di convergenza variazionale. Atti Accad. Naz. Lincei Rend. Cl. Sci. Mat. 58 (1975), 842-850. MR 56 \#6503 Zbl 0339.49005

13. Evans, L. C. \& GARIEPY, R. F. Measure Theory and Fine Properties of Functions. CRC Press, Boca Raton (1992). MR 93f:28001 Zbl 0804.28001

14. Federer, H. Geometric Measure Theory. Springer (1969). MR 41 \#1976|Zbl 0874.49001

15. Fosdick, R. L. \& MASON, D. E. Single phase energy minimizers for materials with nonlocal spatial dependence. Quart. Appl. Math. 53 (1986), 161-195. MR 97a:73085 Zbl 0840.73079

16. KawoHL, B. Rearrangements and Convexity of Level Sets in PDE. Lecture Notes in Math. 1150, Springer, Berlin (1985). MR 87a:35001 Zbl 0593.35002

17. ModicA, L. The gradient theory of phase transitions and the minimal interface criterion. Arch. Rational Mech. Anal. 98 (1987), 123-142. MR 88f:76038ZZbl 0616.76004

18. Modica, L. \& Mortola, S. Un esempio di $\Gamma$-convergenza. Boll. Un. Mat. Ital. Ser. B 14 (1977), 285-299. MR 56 \#3704ZZbl 0356.49008

19. Piccinini, L. C., Stampacchia, G., \& Vidossich, G. Equazioni differenziali ordinarie in $\mathbb{R}^{n}$ (problemi e metodi). Liguori (1978). MR 83k:34002 Zbl 0535.34001

20. Rockafellar, R. T. Convex Analysis. Princeton University Press (1970). MR 97m:49001 Zbl 0193.18401

21. Rogers, R. C. \& Truskinovsky, L. Discretization and hysteresis. Phys. B 233 (1997), 370-375. 